\title{
Analysis of the Risk Sensitive Value Iteration Algorithm
}

\author{
Igor Oliveira Borges $^{1}$, Karina Valdivia Delgado ${ }^{1}$ e Valdinei Freire ${ }^{1}$ \\ ${ }^{1}$ Escola de Artes, Ciências e Humanidades - Universidade de São Paulo (USP) \\ São Paulo, SP - Brasil \\ \{igor.borges,kvd, valdinei.freire\}@usp.br
}

\begin{abstract}
This paper shows an empirical study of Value Iteration Risk Sensitive algorithm proposed by Mihatsch and Neuneier (2002). This approach makes use of a risk factor that allows dealing with different types of risk attitude (prone, neutral or averse) by using a discount factor. We show experiments with the domain of Crossing the River in two different scenarios and we analyze the influence of discount factor and risk factor under two aspects: optimal policy and processing time to convergence. We observed that: (i) the processing cost in extreme risk policies is high with both risk-averse and risk-prone attitude; (ii) a high discount increases time to convergence and reinforces the chosen risk attitude; and (iii) policies with intermediate risk factor values have a low computational cost and show a certain sensitivity to risk based on the discount factor.
\end{abstract}

Keywords. Risk Sensitive Markov Decision Process, Stochastic Planning, Risk Sensitive Policy.

Resumo. Este artigo apresenta um estudo empírico do algoritmo de Iteração de Valor Sensivel a Risco proposto por Mihatsch e Neuneier (2002). Essa abordagem usa um fator de risco que permite lidar com diferentes tipos de risco (propenso, neutro ou averso) e usa fator de desconto. Foram realizados experimentos no domínio de Travessia do Rio em dois cenários de recompensas distintos e feita uma análise da influência do fator de desconto e do fator de risco na política estacionária obtida e no tempo de processamento necessário para convergência nestes cenários. Observou-se que: (i) o custo de processamento de políticas extremas a risco, tanto de aversão quanto de propensão é elevado; (ii) um desconto elevado aumenta o tempo de convergência do algoritmo e reforça a sensibilidade ao risco adotada; e (iii) políticas com valores para o fator de risco intermediários possuem custo computacional baixo e já possuem certa sensibilidade ao risco dependendo do fator de desconto utilizado.

Palavras-Chave. Processo de Decisão Markoviano Sensível a Risco, Planejamento Estocástico, Política Sensível a Risco.

\section{Introdução}

Um modelo comumente utilizado em planejamento probabilístico é o Processo de Decisão Markoviano (em inglês Markovian Decision Process - MDP), neste um agente deve encontrar uma política que minimiza o custo esperado [Puterman 1994].

Um ponto a ser considerado quando da tomada de decisão em planejamento probabilístico é como levar em conta os riscos associados a estocasticidade no resultado de 
decisões. Um agente que minimiza o custo esperado pode ser considerado um agente neutro ao risco, enquanto um agente sensível a risco deve escolher entre duas atitudes: aversa ou propensa ao risco [Shen et al. 2014].

O desenvolvimento de algoritmos sensíveis ao risco, i.e. que consideram a sensibilidade ao risco na tomada de decisão, é um tema pouco explorado na literatura [García and Fernández 2015]. Existem diferentes abordagens para quantificar o risco como: utilidade exponencial esperada [Howard and Matheson 1972, Jaquette 1976, Denardo and Rothblum 1979, Rothblum 1984, Patek 2001], ponderação entre esperança e variância [Sobel 1982, Filar et al. 1989], estimação de desempenho em um intervalo de confiança [Filar et al. 1995, Yu et al. 1998, Hou et al. 2014, Hou et al. 2016].

Nos trabalhos baseados em utilidade exponencial esperada, deve-se especificar um fator de risco, sendo que os valores factíveis para esse fator de risco dependem do problema de decisão em questão [Patek 2001], uma alternativa é considerar fator de desconto, mas nesse caso a política ótima torna-se não estacionária [Chung and Sobel 1987]. [Mihatsch and Neuneier 2002] propõem uma equação de ponto fixo baseado em uma função de transformação escalar por partes e fator de desconto, que permite uma política ótima estacionária como solução, assim como uma escolha arbitrária para o fator de risco.

Embora [Mihatsch and Neuneier 2002] foquem em propor algoritmos de aprendizado por reforço, operadores de ponto-fixo e com propriedade de contração são propostos, o que permite especificar algoritmos de planejamento probabilístico para esse modelo. No entanto, os autores não realizaram nenhuma avaliação empírica desses operadores. Por outro lado, [Freire 2016] explora o papel que o fator de desconto produz em diferentes modelos sensíveis ao risco. Em especial é mostrado que o fator de desconto apresenta uma característica de propensão ao risco quando minimização de custo é considerada.

O objetivo deste trabalho é analisar de forma empírica o impacto da escolha do fator de risco e do fator de desconto sob dois aspectos: (i) quão aversa ao risco é a política obtida sob tais parâmetros, e (ii) como o tempo de convergência do planejamento é afetado por tais parâmetros.

\section{MDPs neutros ao risco}

Processos de decisão markovianos (MDPs) permitem modelar problemas da área de planejamento probabilístico e de aprendizado por reforço. Em MDPs as transições entre estados são definidas probabilisticamente [Puterman 1994] e o processo é chamado de markoviano pois o efeito de uma ação em um dado estado depende somente da ação escolhida naquele estado, não levando em conta o histórico de tomadas de decisão sequenciais já realizadas [Bellman 1957]. Formalmente um MDP é uma tupla: $\langle S, A, T, R\rangle$, no qual:

- $S$ é o conjunto de estados pertencentes ao processo.

- $A$ é o conjunto de ações que podem ser executadas durante as épocas de decisão.

- $T: S \times A \times S \rightarrow[0,1]$ é uma função que define a probabilidade de transição dos estados no sistema, sendo que $T\left(s^{\prime} \mid s, a\right)$ representa a probabilidade de chegar no estado $s^{\prime} \in S$, dado que o agente está no estado $s \in S$ e foi escolhida a ação $a \in A$.

- $R: S \times A \rightarrow R$ é uma função recompensa que define a recompensa recebida no estado $s \in S$ ao tomar uma ação $a \in A$. 
A quantidade de épocas de decisão é chamada de horizonte e pode ser finito (definido por um número fixo), infinito (repetido seguidamente sem parada) ou ainda indeterminado (repetido seguidamente com possibilidade de parada, por exemplo, quando o agente atinge um estado meta).

A solução de um MDP infinito ou indeterminado é uma política estacionária $\pi$, isto é, uma função que mapeia estados em ações; e o valor $V^{\pi}(s)$ de uma política em um estado $s \in S$ é determinado por:

$$
V^{\pi}(s)=\mathrm{E}\left[\sum_{t=0}^{\infty} \gamma^{t} r_{t} \mid \pi, s_{0}=s\right],
$$

em que $\gamma \in[0,1)$ é o fator de desconto.

Seja $\Pi$ o conjunto de políticas estacionárias, a função valor ótima $V^{*}(s)=$ $\max _{\pi \in \Pi} V^{\pi}(s)$ é a solução da equação de Bellman:

$$
V^{*}(s)=\max _{a \in A(s)} \sum_{s^{\prime} \in S} T\left(s^{\prime} \mid s, a\right)\left[R(s, a)+\gamma V^{*}\left(s^{\prime}\right)\right] \forall s \in S .
$$

A política ótima pode ser obtida com base na função valor ótima por:

$$
\pi^{*}(s)=\arg \max _{a \in A(s)} \sum_{s^{\prime} \in S} T\left(s^{\prime} \mid s, a\right)\left[R(s, a)+\gamma V^{*}\left(s^{\prime}\right)\right]
$$

Algoritmo de Iteração de Valor O algoritmo de Iteração de Valor (Value Iteration VI) é um algoritmo de programação dinâmica. Em cada iteração $i$ é calculado o valor $V^{i}(s)$ baseado no valor $V^{i-1}(s)$ para cada estado $s \in S$ do MDP, isto é:

$$
V^{i}(s) \leftarrow \max _{a \in A} \sum_{s^{\prime} \in S} T\left(s^{\prime} \mid s, a\right)\left[R(s, a)+\gamma V^{i-1}\left(s^{\prime}\right)\right]
$$

Um possível critério de parada é considerar o resíduo $\max _{s \in S}\left|V^{i}(s)-V^{i-1}(s)\right|$ e iterar enquanto o resíduo for maior que um erro mínimo desejado $\epsilon$.

\section{MDPs Sensíveis ao Risco}

MDP Sensível a Risco (em inglês Risk Sensitive MDP - RSMDP) é uma extensão de MDP que inclui alguma forma de modelar risco. Neste trabalho estamos interessados na abordagem de [Mihatsch and Neuneier 2002] que está focada principalmente em Aprendizado por Reforço e no lugar de transformar a recompensa acumulada, como é feito na abordagem de utilidade exponencial, a diferença temporal é transformada. Em [Mihatsch and Neuneier 2002] são propostas duas versões com risco dos algoritmos QLearning e Temporal Difference. Além disso, os autores demonstram que os algoritmos propostos convergem e para isso são definidos diferentes operadores. A seguir é descrita a abordagem proposta em [Mihatsch and Neuneier 2002].

Formalmente um RSMDP é definido por uma tupla $\langle$ MDP, $k, \gamma\rangle$, sendo $-1<k<$ 1 o fator de risco e $\gamma \in[0,1)$ o fator de desconto. Seja a função de transformação $\mathcal{X}^{k}$ que depende do sinal da entrada $x$ e do fator de risco, definida por: 


$$
\mathcal{X}^{k}(x)= \begin{cases}(1-k) x & \text { se } x>0 \\ (1+k) x & \text { caso contrário }\end{cases}
$$

Dada uma política estacionária $\pi$, a função valor $V_{k}^{\pi}(s)$ correspondente pode ser obtida resolvendo o seguinte sistema de equações para todo $s \in S$ :

$$
0=\sum_{s^{\prime} \in S} T\left(s^{\prime} \mid s, \pi(s)\right) \chi^{k}\left(R(s, \pi(s))+\gamma V_{k}^{\pi}\left(s^{\prime}\right)-V_{k}^{\pi}(s)\right)
$$

Seja $x=R(s, \pi(s))+\gamma V_{k}^{\pi}\left(s^{\prime}\right)-V_{k}^{\pi}(s)$ a diferença temporal, se $k$ for positivo, então diferenças temporais negativas tem peso de ponderação maior que as positivas. Em outras palavras se: $R(s, \pi(s))+\gamma V_{k}^{\pi}\left(s^{\prime}\right)-V_{k}^{\pi}(s)<0$, transições para estados sucessores em que a recompensa imediata passou a ser menor que a média recebem um peso extra maior. Enquanto, as transições para estados que prometem um retorno maior do que a média recebem uma ponderação menor. Ou seja, se $k>0$, a função objetivo $V_{k}^{\pi}(s)$ é aversa ao risco e é propensa ao risco, se $k<0$, em $k=0$ é neutra a risco e existe a equivalência com o critério de MDPs clássicos. No limite de extrema aversão ao risco, quando $k \rightarrow 1$, a função objetivo resolve um problema equivalente a otimização no pior dos casos. No limite de extrema propensão a risco, quando $k \rightarrow-1$, o agente é muito otimista assumindo que para todos os possíveis próximos estados, aquele que acontece é sempre o melhor.

Teorema 1 (Solução única e casos limites) [Mihatsch and Neuneier 2002] Para cada $k \in(-1,1)$ existe uma solução única $V_{k}^{\pi}(s)$ obtida pela resolução da Equação 2. Assim a função valor sensível a risco está bem definida. Para $k=0, k \rightarrow 1$ e $k \rightarrow-1$, temos:

$$
\begin{gathered}
V_{0}^{\pi}(s)=E\left(\sum_{t=0}^{\infty} \gamma^{t} \mathcal{R}\left(s_{t}, \pi\left(s_{t}\right)\right) \mid s_{0}=s\right), \\
\lim _{k \rightarrow 1} V_{k}^{\pi}(s)=\inf \left(\sum_{t=0}^{\infty} \gamma^{t} \mathcal{R}\left(s_{t}, \pi\left(s_{t}\right)\right) \mid s_{0}=s\right), \\
\lim _{k \rightarrow-1} V_{k}^{\pi}(s)=\sup \left(\sum_{t=0}^{\infty} \gamma^{t} \mathcal{R}\left(s_{t}, \pi\left(s_{t}\right)\right) \mid s_{0}=s\right) .
\end{gathered}
$$

O teorema mostra que a abordagem permite interpolar entre os critérios de melhor caso, neutro e pior caso. Uma política estacionária $\pi^{*}$ é ótima, se $V_{k}^{\pi^{*}}(s) \geq V_{k}^{\pi}(s)$ $\forall \pi \in \Pi, s \in S$.

\subsection{Operador Sensível a Risco}

Os Lemas 1 e 2 apresentados nesta seção foram adaptados de [Mihatsch and Neuneier 2002].

Lema 1 Seja $k \in(-1,1)$. Para cada par de números reais a, b, existe uma $\xi_{(a, b, k)} \in$ $[1-|k|, 1+|k|]$ tal que $\chi^{k}(a)-\chi^{k}(b)=\xi_{(a, b, k)}(a-b)$. 
Em [Mihatsch and Neuneier 2002] foram definidos diferentes operadores, entre eles, o operador $\mathcal{N}_{\alpha, k}$ sobre o espaço de funções $Q(s, a)$, tal que:

$$
\mathcal{N}_{\alpha, k}[Q](s, a):=Q(s, a)+\alpha \sum_{s^{\prime} \in S} T\left(s^{\prime} \mid s, a\right) \chi^{k}\left(R(s, a)+\gamma \max _{u \in A(s)} Q\left(s^{\prime}, u\right)-Q(s, a)\right),
$$

em que $\alpha$ denota um tamanho de passo positivo. Esse operador é um mapeamento de contração relacionado à norma máxima $|Q|:=\max _{s \in S, a \in A(s)}|Q(s, a)|$ desde que $\alpha$ seja pequeno o suficiente, como especificado no próximo lema.

Lema 2 Seja $k \in(-1,1), 0 \leq \gamma<1$ e $0<\alpha \leq(1+|k|)^{-1}$ para todas as funções $Q_{1} e$ $Q_{2}$, é verdade que:

$$
\left|\mathcal{N}_{\alpha, k}\left[Q_{1}\right]-\mathcal{N}_{\alpha, k}\left[Q_{2}\right]\right| \leq \rho\left|Q_{1}-Q_{2}\right|,
$$

em que $\rho=(1-\alpha(1-|k|)(1-\gamma)) \in(0,1)$. Assim o operador $\mathcal{N}_{\alpha, k}$ é um mapeamento de contração.

Note que $\alpha$ está definido em $0<\alpha \leq(1+|k|)^{-1}$, resultado obtido com este estudo. Para garantir que $\alpha$ possa ser igual a $(1+|k|)^{-1}$, basta definir que o desconto $\gamma$ seja necessariamente inferior a 1.

\section{Algoritmo de Iteração de Valor Sensível a Risco}

A partir da Equação 6 e do Lema 2, é possível definir a função de atualização de $Q$ a seguir, a qual é utilizada no algoritmo de Iteração de Valor Sensível a Risco:

$$
Q^{i}(s, a) \leftarrow Q^{i-1}(s, a)+\alpha \sum_{s^{\prime} \in \mathcal{S}} \mathcal{T}\left(s^{\prime} \mid s, a\right) \mathcal{X}^{k}\left(\mathcal{R}(s, a)+\gamma \max _{a} Q^{i-1}\left(s^{\prime}, a\right)-Q^{i-1}(s, a)\right)
$$

Nessa equação, a função escalar $\mathcal{X}^{k}$ é aplicada também diretamente nas diferenças temporais dos valores. $\mathrm{O}$ fator $\alpha$ é usado para garantir convergência e segundo o Lema 2, os valores possíveis são $0<\alpha \leq(1+|k|)^{-1}$. De maneira intuitiva, o fator $\alpha$ assegura que os valores parciais não cresçam muito, em especial quando o fator de desconto $\gamma$ estiver próximo de 1 e o fator de risco $k$ for negativo.

Dado $Q^{i}(s, a)$, é possível obter a função valor na iteração $i$ :

$$
V^{i}(s)=\max _{a}\left\{Q^{i}(s, a)\right\}
$$

e também é possível obter uma política gulosa:

$$
\pi_{\chi^{k}}(s)=\arg \max _{a}\left\{Q^{i}(s, a)\right\} .
$$

O critério de parada usado no algoritmo de Iteração de Valor Sensível a Risco é baseado no seguinte residual relativo:

$$
\operatorname{residual}(s)=\left|\frac{V^{i}(s)-V^{i-1}(s)}{V^{i-1}(s)}\right|
$$


Dado um erro mínimo desejado $\epsilon$, se $\max _{s \in \mathcal{S}}\{\operatorname{residual}(s)\} \leq \epsilon$, o algoritmo para. Note que a utilização do residual relativo é melhor para o algoritmo de Iteração de Valor Sensível a Risco do que o residual absoluto uma vez que o algoritmo pode ter valores muito diferentes para $V^{i}(s)$ e $V^{i-1}(s)$.

O algoritmo de Iteração de Valor Sensível a Risco (Algoritmo 1) recebe como entrada um RSMDP, o erro mínimo desejado $\epsilon$ e o fator $\alpha$; e devolve como saída a função valor V ótima sensível a risco. Nas linhas 1 a 4 são inicializados o valor $V^{0}$ com a maior recompensa para cada estado e o valor $Q_{0}$ com 0 para todo par estado-ação. Em cada iteração $i$ o algoritmo atualiza os valores usando a Equação 7 (Linha 9). Na linha 11, $V^{i}$ é calculada com base em $Q^{i}$ e na linha 12 é calculado o residual relativo entre as iterações $i$ e $i-1$ de cada estado. Por fim, na linha 16 o algoritmo devolve a função valor $V$.

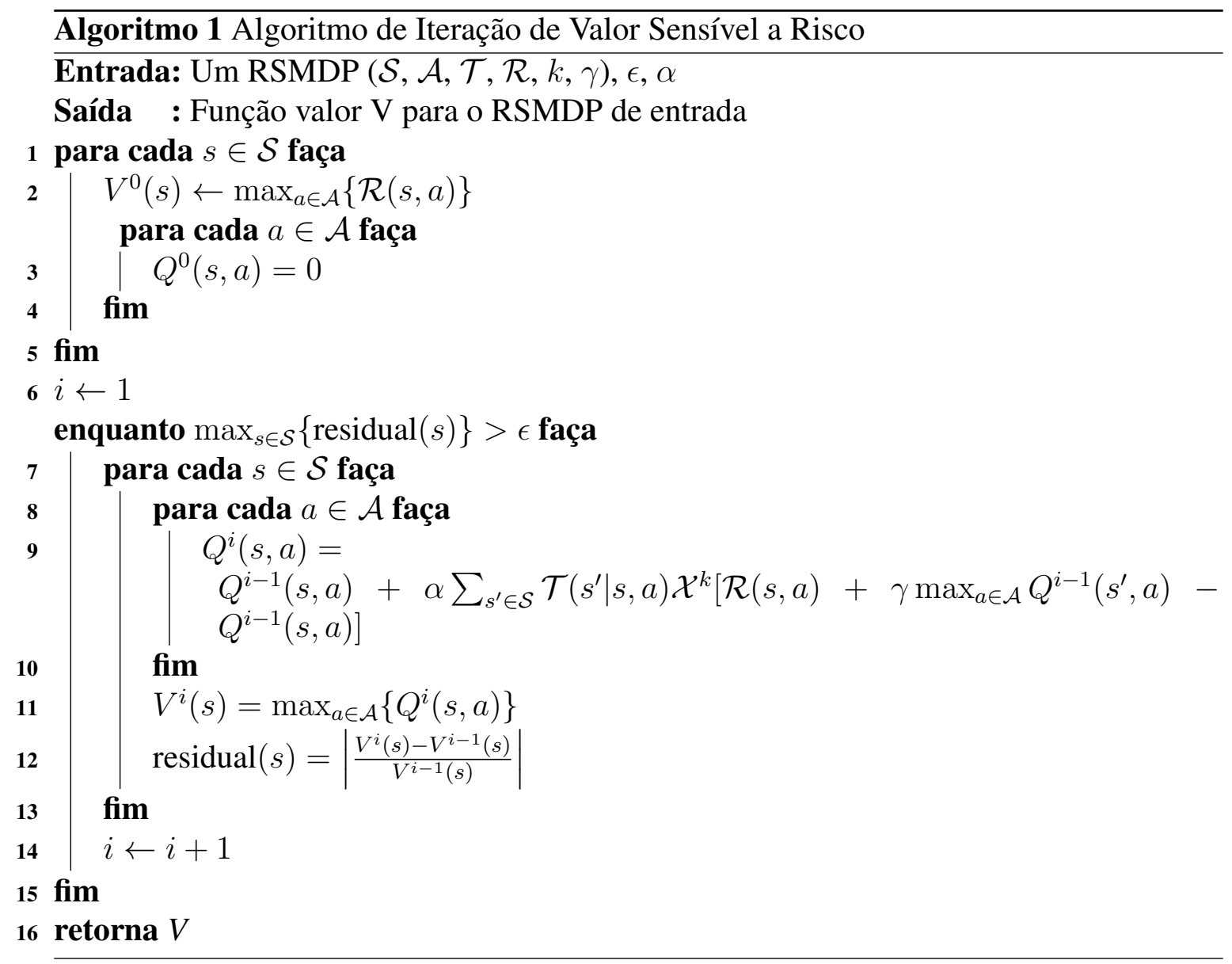

\section{Experimentos}

Nesta seção analisamos o algoritmo de Iteração de Valor Sensível a Risco no domínio de Travessia do Rio. O código-fonte ${ }^{1}$ que inclui a modelagem do domínio do rio, o algoritmo de iteração de valor sensível a risco e a interface gráfica foi desenvolvido em Octave/Matlab. Os experimentos foram realizados no Matlab R2015a, na versão acadêmica de 64 bits no Windows 10, em um processador Intel Core i7-3537U CPU @ 3.10Ghz, 8 GB de memória RAM @ 1600Mhz, 256 GB de armazenamento SSD. A interface gráfica foi desabilitada para o experimento.

\footnotetext{
${ }^{1}$ Disponível em: https://github.com/RSVI
} 


\subsection{Domínio de Travessia no Rio}

O problema da Travessia do Rio [Freire and Delgado 2017] é representado como um grid de tamanho $N_{x} \times N_{y}$. Um exemplo pode ser visualizado na Tabela 1. Nesta tabela está em azul claro, o rio; em azul escuro, a cachoeira; em vermelho, a ponte; em verde, o solo; em cinza, o agente propriamente dito no estado inicial; e por fim a meta é destacada em amarelo.

O domínio consiste em apenas um agente no extremo do canto inferior esquerdo da matriz, o qual tem como objetivo chegar ao canto inferior direito do grid, sendo que apenas 4 movimentos são possíveis: ir para o norte $(N)$, sul $(S)$, leste $(E)$, e oeste $(W)$. Assim o agente pode chegar no objetivo de duas formas: (i) nadando a partir de qualquer ponto do rio; ou (ii) subindo o grid até a ponte que está na posição extrema superior.

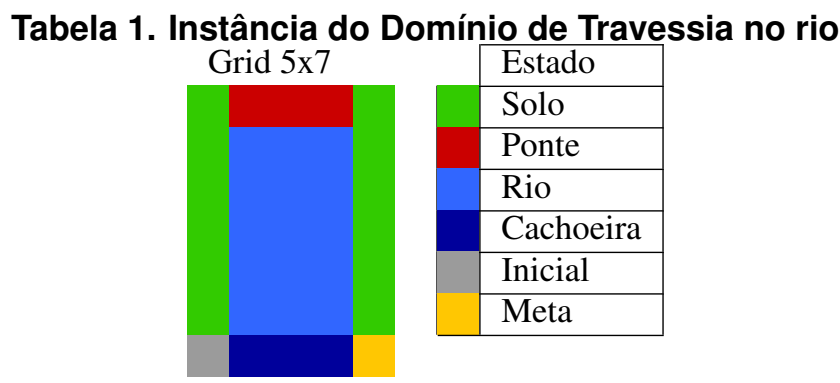

\subsection{Configurações do Experimento}

Para o experimento adotou-se a condição de parada $\epsilon=0.00001$; fator de desconto $\gamma \in$ $\{0.6 ; 0.7 ; 0.8 ; 0.9 ; 0.99\}$; e fator de risco $k \in\{-0.99 ;-0.8 ;-0.5 ; 0 ; 0.5 ; 0.8 ; 0.99\}$.

A probabilidade de transição fora do rio é de $99 \%$ da ação ter o efeito desejado e $1 \%$ de ficar parado. No rio, o agente tem $20 \%$ de chance de ser arrastado pela correnteza e $80 \%$ de sucesso na execução da ação escolhida. A probabilidade de ir para o estado inicial dado que o agente está na cachoeira é 1, isto é, sempre que o agente cai na cachoeira ele retorna para o estado inicial.

Os experimentos foram realizados em dois cenários de recompensa distintos, $\mathrm{o}$ primeiro com recompensa 0 em cada estado e +1 no estado meta, o qual chamaremos de cenário de recompensas acumuladas positivas $(+)$; e o segundo com recompensa negativa -1 em cada estado e 0 na meta, o qual chamaremos de cenário de recompensas acumuladas negativas ( - ). Além disso, foram realizados experimentos com $\alpha=0.5$ e com $\alpha$ definido baseado no valor de $k$, como mostrado na Tabela 2.

Tabela 2. Relação de valor entre fator de risco $k$ e fator $\alpha$ adotado no experimento.

\begin{tabular}{|c|c|}
\hline$k$ & $\alpha$ \\
\hline-0.99 ou 0.99 & 0.50 \\
\hline-0.8 ou 0.8 & 0.56 \\
\hline-0.5 ou 0.5 & 0.67 \\
\hline 0 & 1 \\
\hline
\end{tabular}




\subsection{Políticas Obtidas}

Foram realizados experimentos com diferentes tamanhos de grid, entre eles $3 \times 7,5 \times 7$, $7 \times 7$ e $10 \times 7$. Nesta seção são avaliados os resultados obtidos para o grid $10 \times 7$ pois ele apresenta uma variedade maior de políticas. A Tabela 3 mostra as políticas obtidas para esse grid, variando $k$ e $\gamma$ no algoritmo de Iteração de Valor sensível a risco nos cenário de recompensa $(+)$ e recompensa $(-)$, parte superior e inferior da tabela, respectivamente. Tanto ao adotar $\alpha=0.5$ quanto com o $\alpha$ relativo a $k$, foram obtidas as mesmas políticas relativas ao cenário de recompensa adotado, isto ocorre pois para ambos os valores de $\alpha$ utilizados, é garantida a convergência pelo Lema 2.

Em ambos cenários se observa um comportamento de risco esperado para os parâmetros de propensão, neutralidade e aversão ao risco, sendo que para $k=-0.8$, $k=-0.5, k=0, k=0.5$ e $k=0.8$ as políticas encontradas variando $\gamma$ são as mesmas. A diferença entre ambos cenários acontece nos extremos, com $k=-0.99$ e $k=0.99$.

Para $k=-0.99$, no cenário $(-)$ há algumas políticas com atitudes mais propensas ao risco que não aparecem no cenário de recompensa $(+)$, em especial saltar da ponte a fim de chegar mais rápido na meta (veja no cenário de recompensa acumulada negativa $k=-0.99$ e $\gamma \geq 0.7$ ). Para $k=0.99$ aparecem duas políticas diferentes no cenário $(-)$ e no cenário positivo três políticas diferentes. Nessas políticas o agente tenta atravessar pela ponte e caso caia no rio tenta voltar a borda mais perto ou se está perto da ponte tenta subir para ter uma travessia mais segura evitando cair na cachoeira.

Em valores de $k \geq-0.5$, observasse políticas mais conservadoras que tentam atravessar pela ponte e evitam entrar no rio para assegurar uma transição mais segura. Ao tomar uma política mais propensa $(k=-0.99$ e $k=-0.8)$, o agente tende a se arrisca mais atravessando pelo rio e saltando da ponte em direção a meta.

Nota-se ainda que nos extremos de valor de risco $k=-0.99$ para propensão e $k=0.99$ para aversão obtém-se políticas mais reforçadas para o respectivo tipo de atitude esperada, em especial quando o $\gamma$ é elevado $(\gamma \geq 0.9)$. Note que o fator de desconto tem o papel de atenuar as atitudes se o desconto for pequeno ou realçar as atitudes se o desconto for grande. O parâmetro de risco por sua vez infere na política obtida um comportamento de propensão, neutralidade ou aversão conforme o valor do parâmetro escolhido.

\subsection{Tempo de Processamento}

As Figuras 1 e 2 apresentam o tempo de processamento para o cenário de recompensas $(+)$ e $(-)$ com duas configurações de $\alpha$ distintas $(\alpha=0.5$ e $\alpha$ relativo a $k$ ) no grid $10 \times 7$. Como comentado anteriormente as políticas obtidas para $\alpha$ fixo e $\alpha$ relativo para cada cenário de recompensa adotado foram iguais entre si. Porém, o tempo de processamento necessário para a convergência foi diferente, sendo mais eficiente escolher um fator $\alpha$ relativo ao fator de risco escolhido, especialmente para $k=0$.

No grid $10 \times 7, \gamma=0.99$ e $\alpha$ relativo, o tempo necessário para convergência para $k=-0.8$ e $k=0.8$ é aproximadamente $4 s$ e $12 s$ no cenário $(+)$ e de $9 s$ e $10 s$ no cenário $(-)$, respectivamente. Enquanto no intervalo de risco $-0.5 \leq k \leq 0.5$ notou-se uma execução rápida, independente do fator de desconto adotado. Para valores extremos de $k(k=-0.99$ e $k=0.99)$ têm-se uma maior demanda de tempo para processamento quando comparado com outros valores de $k$. 
Tabela 3. Políticas obtidas pelo algoritmo de Iteração de Valor Sensível a Risco em um grid $10 \times 7$ conforme cenário de recompensa adotado.

\begin{tabular}{|c|c|c|c|c|c|c|c|}
\hline $10 \times 7(+)$ & $\mathrm{k}=-0.99$ & $\mathrm{k}=-0.8$ & $\mathrm{k}=-0.5$ & $\mathrm{k}=0$ & $\mathrm{k}=0.5$ & $\mathrm{k}=0.8$ & $\mathrm{k}=0.99$ \\
\hline \multirow[b]{7}{*}{$\gamma=0.6$} & & $\rightarrow \rightarrow \rightarrow \rightarrow \rightarrow \rightarrow \rightarrow \rightarrow 1$ & $\rightarrow \rightarrow \rightarrow \rightarrow \rightarrow \rightarrow \rightarrow \rightarrow \rightarrow 1$ & $\rightarrow \rightarrow \rightarrow \rightarrow \rightarrow \rightarrow \rightarrow \rightarrow \rightarrow 1$ & $\rightarrow \rightarrow \rightarrow \rightarrow \rightarrow \rightarrow \rightarrow \rightarrow \rightarrow 1$ & $\rightarrow \rightarrow \rightarrow \rightarrow \rightarrow \rightarrow \rightarrow \rightarrow \rightarrow 1$ & $\rightarrow \rightarrow \rightarrow \rightarrow \rightarrow \rightarrow \rightarrow \rightarrow \rightarrow 1$ \\
\hline & $\rightarrow \rightarrow \rightarrow \rightarrow \rightarrow \rightarrow \rightarrow \rightarrow \downarrow$ & $\rightarrow \rightarrow \rightarrow \rightarrow \rightarrow \rightarrow \rightarrow 1$ & $\uparrow \uparrow \uparrow \uparrow \rightarrow \rightarrow \rightarrow \rightarrow \downarrow$ & $\uparrow \uparrow \uparrow \uparrow \uparrow \uparrow \uparrow \rightarrow \rightarrow \downarrow$ & $\uparrow \uparrow \uparrow \uparrow \uparrow \uparrow \uparrow \uparrow \rightarrow \downarrow$ & $\uparrow \uparrow \uparrow \uparrow \uparrow \uparrow \uparrow \uparrow \rightarrow \downarrow$ & $\uparrow \uparrow \uparrow \uparrow \uparrow \uparrow \uparrow \uparrow \rightarrow \downarrow$ \\
\hline & $\rightarrow \rightarrow \rightarrow \rightarrow \rightarrow \rightarrow \rightarrow \rightarrow 1$ & $\rightarrow \rightarrow \rightarrow \rightarrow \rightarrow \rightarrow \rightarrow \rightarrow 1$ & $\uparrow \rightarrow \rightarrow \rightarrow \rightarrow \rightarrow \rightarrow \rightarrow$ & $\uparrow \uparrow \uparrow \uparrow \rightarrow \rightarrow \rightarrow \rightarrow \downarrow$ & $\uparrow+\uparrow \uparrow \uparrow \uparrow \rightarrow \rightarrow \rightarrow \downarrow$ & $\uparrow \leftarrow \uparrow \uparrow \uparrow \uparrow \rightarrow \rightarrow \rightarrow \downarrow$ & $\uparrow \leftarrow \uparrow \uparrow \uparrow \uparrow \rightarrow \rightarrow \rightarrow \downarrow$ \\
\hline & $\rightarrow \rightarrow \rightarrow \rightarrow \rightarrow \rightarrow \rightarrow \rightarrow \downarrow$ & $\rightarrow \rightarrow \rightarrow \rightarrow \rightarrow \rightarrow \rightarrow \rightarrow 1$ & $\uparrow \rightarrow \rightarrow \rightarrow \rightarrow \rightarrow \rightarrow \downarrow$ & $\uparrow \mapsto \uparrow \rightarrow \rightarrow \rightarrow \rightarrow \rightarrow 1$ & $\uparrow \leftrightarrow \mapsto \uparrow \rightarrow \rightarrow \rightarrow \rightarrow \downarrow$ & $\uparrow \leftarrow \leftarrow \rightarrow \rightarrow \rightarrow \rightarrow \rightarrow \downarrow$ & $\uparrow \leftrightarrow \leftrightarrow \rightarrow \rightarrow \rightarrow \rightarrow \downarrow$ \\
\hline & $\rightarrow \rightarrow \rightarrow \rightarrow \rightarrow \rightarrow \rightarrow \rightarrow \rightarrow 1$ & $\rightarrow \rightarrow \rightarrow \rightarrow \rightarrow \rightarrow \rightarrow \rightarrow 1$ & $\uparrow \rightarrow \rightarrow \rightarrow \rightarrow \rightarrow \rightarrow \downarrow$ & $\uparrow \leftrightarrow \leftrightarrow \rightarrow \rightarrow \rightarrow \rightarrow \downarrow$ & $\uparrow \leftrightarrow \leftrightarrow \rightarrow \rightarrow \rightarrow \rightarrow \rightarrow \downarrow$ & $\uparrow \hookleftarrow \leftarrow \rightarrow \rightarrow \rightarrow \rightarrow \downarrow$ & $\uparrow \leftrightarrow \leftrightarrow \rightarrow \rightarrow \rightarrow \rightarrow \downarrow$ \\
\hline & & $\uparrow \rightarrow \rightarrow \rightarrow \rightarrow \rightarrow \rightarrow \rightarrow$ & $\uparrow \rightarrow \rightarrow \rightarrow \rightarrow \rightarrow \rightarrow \rightarrow$ & $\uparrow \leftrightarrow \leftrightarrow \rightarrow \rightarrow \rightarrow \rightarrow \downarrow$ & $\uparrow \leftrightarrow \leftrightarrow \rightarrow \rightarrow \rightarrow \rightarrow \downarrow$ & $\uparrow \hookleftarrow \leftrightarrow \rightarrow \rightarrow \rightarrow \rightarrow \downarrow$ & $\uparrow \leftrightarrow \leftrightarrow \rightarrow \rightarrow \rightarrow \rightarrow$ \\
\hline & $\uparrow+++++++++0$ & $\uparrow++++++++0$ & $\uparrow++++++++0$ & $\uparrow++++++++0$ & $\uparrow++++++++0$ & $\uparrow++++++++0$ & $\uparrow++++++++0$ \\
\hline \multirow[b]{7}{*}{$\gamma=0.7$} & $\rightarrow \rightarrow \rightarrow \rightarrow \rightarrow \rightarrow \rightarrow \downarrow \downarrow$ & $\rightarrow \rightarrow \rightarrow \rightarrow \rightarrow \rightarrow \rightarrow$ & $\rightarrow \rightarrow \rightarrow \rightarrow \rightarrow \rightarrow \rightarrow \rightarrow \rightarrow \mid$ & $\rightarrow \rightarrow \rightarrow \rightarrow \rightarrow \rightarrow \rightarrow \rightarrow \mid$ & $\rightarrow \rightarrow \rightarrow \rightarrow \rightarrow \rightarrow \rightarrow \rightarrow \downarrow$ & $\rightarrow \rightarrow \rightarrow \rightarrow \rightarrow \rightarrow \rightarrow \rightarrow \rightarrow \downarrow$ & $\rightarrow \rightarrow \rightarrow \rightarrow \rightarrow \rightarrow \rightarrow \rightarrow \mid$ \\
\hline & $\rightarrow \rightarrow 1$ & $\rightarrow \rightarrow \rightarrow \rightarrow \rightarrow \rightarrow \rightarrow \rightarrow 1$ & $\uparrow \uparrow \uparrow \uparrow \uparrow \rightarrow \rightarrow \rightarrow \downarrow$ & $\uparrow \uparrow \uparrow \uparrow \uparrow \uparrow \uparrow \rightarrow \rightarrow \downarrow$ & $\uparrow \uparrow \uparrow \uparrow \uparrow \uparrow \uparrow \uparrow \rightarrow \downarrow$ & $\uparrow \uparrow \uparrow \uparrow \uparrow \uparrow \uparrow \uparrow \rightarrow \downarrow$ & $\uparrow \uparrow \uparrow \uparrow \uparrow \uparrow \uparrow \uparrow \rightarrow \downarrow$ \\
\hline & & $\rightarrow \rightarrow \rightarrow \rightarrow \rightarrow \rightarrow \rightarrow \rightarrow 1$ & $\uparrow \uparrow \rightarrow \rightarrow \rightarrow \rightarrow \rightarrow \rightarrow \downarrow$ & $\uparrow \leftrightarrow \uparrow \uparrow \uparrow \rightarrow \rightarrow \rightarrow \downarrow$ & $\uparrow \uparrow \uparrow \uparrow \uparrow \uparrow \rightarrow \rightarrow \downarrow \downarrow$ & $\uparrow \leftarrow \uparrow \uparrow \uparrow \uparrow \rightarrow \rightarrow \rightarrow \downarrow$ & $\uparrow \uparrow \uparrow \uparrow \uparrow \uparrow \rightarrow \rightarrow \rightarrow$ \\
\hline & $\rightarrow \rightarrow$ & $\rightarrow \rightarrow \rightarrow \rightarrow \rightarrow \rightarrow \rightarrow \rightarrow 1$ & $\uparrow \leftrightarrow \rightarrow \rightarrow \rightarrow \rightarrow \rightarrow \downarrow$ & $\uparrow+\uparrow \rightarrow \rightarrow \rightarrow \rightarrow \rightarrow \downarrow$ & $\uparrow \leftrightarrow \leftrightarrow \leftrightarrow \rightarrow \rightarrow \rightarrow \downarrow$ & $\uparrow \hookleftarrow \leftrightarrow \rightarrow \rightarrow \rightarrow \rightarrow \downarrow$ & $\uparrow \leftrightarrow \leftrightarrow \leftrightarrow \rightarrow \rightarrow \rightarrow \rightarrow \downarrow$ \\
\hline & & $\rightarrow \rightarrow \rightarrow \rightarrow \rightarrow \rightarrow \rightarrow 1$ & $\uparrow \leftrightarrow \rightarrow \rightarrow \rightarrow \rightarrow \rightarrow \downarrow$ & $\uparrow \leftrightarrow \leftrightarrow \rightarrow \rightarrow \rightarrow \rightarrow \downarrow$ & $\uparrow \mapsto \leftrightarrow \rightarrow \rightarrow \rightarrow \rightarrow \downarrow$ & $\uparrow \hookleftarrow \leftarrow \rightarrow \rightarrow \rightarrow \rightarrow \rightarrow \downarrow$ & $\uparrow \leftrightarrow \leftrightarrow \leftrightarrow \rightarrow \rightarrow \rightarrow \rightarrow \downarrow$ \\
\hline & & $\uparrow \uparrow \uparrow \rightarrow \rightarrow \rightarrow \rightarrow \rightarrow \downarrow$ & $\uparrow \leftrightarrow \rightarrow \rightarrow \rightarrow \rightarrow \rightarrow \downarrow$ & $\uparrow \leftrightarrow \leftrightarrow \rightarrow \rightarrow \rightarrow \rightarrow \mid$ & $\uparrow \leftrightarrow \leftrightarrow \leftrightarrow \leftrightarrow \rightarrow \rightarrow \downarrow$ & $\uparrow \hookleftarrow \leftrightarrow \rightarrow \rightarrow \rightarrow \rightarrow \downarrow$ & $\uparrow \leftrightarrow \leftrightarrow \rightarrow \rightarrow \rightarrow \rightarrow \downarrow$ \\
\hline & $\uparrow++++++++0$ & $\uparrow++++++++0$ & $\uparrow++++++++0$ & $\uparrow++++++++0$ & $\uparrow++++++++0$ & $\uparrow++++++++0$ & $\uparrow++++++++0$ \\
\hline \multirow[b]{7}{*}{$\gamma=0.8$} & $\rightarrow \downarrow \downarrow$ & $\rightarrow \rightarrow \rightarrow \rightarrow \rightarrow \rightarrow \rightarrow \rightarrow \mid$ & $\rightarrow \rightarrow \rightarrow \rightarrow \rightarrow \rightarrow \rightarrow \rightarrow \rightarrow 1$ & $\rightarrow \rightarrow \rightarrow \rightarrow \rightarrow \rightarrow \rightarrow \rightarrow \mid$ & $\rightarrow \rightarrow \rightarrow \rightarrow \rightarrow \rightarrow \rightarrow \rightarrow \mid$ & $\rightarrow \rightarrow \rightarrow \rightarrow \rightarrow \rightarrow \rightarrow \rightarrow \rightarrow \downarrow$ & $\rightarrow \rightarrow \rightarrow \rightarrow \rightarrow \rightarrow \rightarrow \rightarrow \mid$ \\
\hline & $\rightarrow \downarrow$ & $\rightarrow \rightarrow \rightarrow \rightarrow$ & $\uparrow \uparrow \uparrow \uparrow \uparrow \rightarrow \rightarrow \rightarrow \rightarrow \downarrow$ & $\uparrow \uparrow \uparrow \uparrow \uparrow \uparrow \uparrow \rightarrow \rightarrow \downarrow$ & $\uparrow \uparrow \uparrow \uparrow \uparrow \uparrow \uparrow \uparrow \rightarrow \downarrow$ & $\uparrow \uparrow \uparrow \uparrow \uparrow \uparrow \uparrow \uparrow \rightarrow \downarrow$ & $\uparrow \uparrow \uparrow \uparrow \uparrow \uparrow \uparrow \uparrow \rightarrow \downarrow$ \\
\hline & $\rightarrow \downarrow$ & $\rightarrow \rightarrow \rightarrow \rightarrow$ & $\uparrow \uparrow \uparrow \rightarrow \rightarrow \rightarrow \rightarrow \rightarrow \downarrow$ & $\uparrow \leftarrow \uparrow \uparrow \uparrow \rightarrow \rightarrow \rightarrow \rightarrow$ & $\uparrow+\uparrow \uparrow \uparrow \uparrow \rightarrow \rightarrow \rightarrow \downarrow$ & $\uparrow \leftarrow \leftarrow \uparrow \uparrow \uparrow \uparrow \rightarrow \rightarrow \downarrow$ & $\uparrow \leftarrow \uparrow \uparrow \uparrow \uparrow \rightarrow \rightarrow \downarrow$ \\
\hline & & $\rightarrow 1$ & $\uparrow \leftrightarrow \rightarrow \rightarrow \rightarrow \rightarrow \downarrow$ & $\uparrow \leftarrow \uparrow \rightarrow \rightarrow \rightarrow \rightarrow \downarrow$ & $\uparrow \leftrightarrow \leftrightarrow \rightarrow \rightarrow \rightarrow \rightarrow \downarrow$ & $\uparrow \leftrightarrow \leftrightarrow \rightarrow \rightarrow \rightarrow \rightarrow \downarrow$ & $\uparrow \leftrightarrow \leftrightarrow \leftrightarrow \rightarrow \rightarrow \rightarrow \downarrow$ \\
\hline & & $\uparrow \rightarrow \rightarrow \rightarrow \rightarrow$ & $\uparrow \leftrightarrow \leftrightarrow \rightarrow \rightarrow \rightarrow \rightarrow \rightarrow 1$ & $\uparrow \leftrightarrow \leftrightarrow \rightarrow \rightarrow \rightarrow \rightarrow 1$ & $\uparrow \leftrightarrow \leftrightarrow \rightarrow \rightarrow \rightarrow \rightarrow \perp$ & $\uparrow \hookleftarrow \leftrightarrow \rightarrow \rightarrow \rightarrow \rightarrow 1$ & $\uparrow \leftrightarrow \leftrightarrow \rightarrow \rightarrow \rightarrow \rightarrow 1$ \\
\hline & $\rightarrow \rightarrow \rightarrow \rightarrow \rightarrow \rightarrow \rightarrow \rightarrow \rightarrow 1$ & $\uparrow \uparrow \uparrow \uparrow \uparrow \uparrow-$ & $\uparrow \leftrightarrow \leftrightarrow \rightarrow \rightarrow \rightarrow \rightarrow \downarrow$ & $\uparrow \leftrightarrow \leftrightarrow \rightarrow \rightarrow \rightarrow \downarrow$ & $\uparrow \leftrightarrow \leftrightarrow \leftrightarrow \rightarrow \rightarrow \rightarrow \downarrow$ & $\uparrow \leftrightarrow \leftrightarrow \rightarrow \rightarrow \rightarrow \rightarrow \downarrow$ & $\uparrow \leftrightarrow \leftrightarrow \rightarrow \rightarrow \rightarrow \rightarrow$ \\
\hline & $\uparrow++++++++0$ & $1++++++++0$ & $\uparrow++++++++0$ & $\uparrow++++++++0$ & $\uparrow++++++++0$ & $\uparrow++++++++0$ & $\uparrow++++++++0$ \\
\hline \multirow[b]{6}{*}{$\gamma=0.9$} & $\rightarrow \downarrow$ & $\rightarrow \rightarrow \rightarrow \rightarrow \rightarrow$ & $\rightarrow \rightarrow \rightarrow \rightarrow \rightarrow \rightarrow \rightarrow \rightarrow \mid$ & $\rightarrow \rightarrow \rightarrow \rightarrow \rightarrow \rightarrow \rightarrow \rightarrow \downarrow$ & $\rightarrow \rightarrow \rightarrow \rightarrow \rightarrow \rightarrow \rightarrow \rightarrow \rightarrow \mid$ & $\rightarrow \rightarrow \rightarrow \rightarrow \rightarrow \rightarrow \rightarrow \rightarrow \downarrow$ & $\rightarrow \rightarrow \rightarrow \rightarrow \rightarrow \rightarrow \rightarrow \rightarrow 1$ \\
\hline & & $\uparrow \rightarrow \rightarrow \rightarrow-$ & $\uparrow \uparrow \uparrow \uparrow \uparrow \uparrow \rightarrow \rightarrow \rightarrow \downarrow$ & $\uparrow \uparrow \uparrow \uparrow \uparrow \uparrow \uparrow \uparrow \rightarrow \downarrow$ & $\uparrow \uparrow \uparrow \uparrow \uparrow \uparrow \uparrow \uparrow \uparrow \rightarrow \downarrow$ & $\uparrow \uparrow \uparrow \uparrow \uparrow \uparrow \uparrow \uparrow \rightarrow \downarrow$ & $\uparrow \uparrow \uparrow \uparrow \uparrow \uparrow \uparrow \uparrow \rightarrow \downarrow$ \\
\hline & $\rightarrow \rightarrow \downarrow$ & $\uparrow \rightarrow \rightarrow \rightarrow$ & $\uparrow \uparrow \uparrow \uparrow \uparrow \rightarrow \rightarrow \rightarrow \downarrow$ & $\uparrow \uparrow \uparrow \uparrow \uparrow \uparrow \rightarrow \rightarrow \downarrow$ & $\uparrow \hookleftarrow \leftarrow \uparrow \uparrow \uparrow \rightarrow \rightarrow \rightarrow \downarrow$ & $\uparrow \leftrightarrow \leftrightarrow \rightarrow \rightarrow \rightarrow \downarrow$ & $\uparrow \leftrightarrow \leftrightarrow \leftrightarrow \rightarrow \rightarrow \rightarrow 1$ \\
\hline & $\rightarrow \rightarrow \rightarrow \downarrow$ & $\uparrow \rightarrow \rightarrow-$ & $\uparrow \leftrightarrow \leftrightarrow \rightarrow \rightarrow \rightarrow \rightarrow \downarrow$ & $\uparrow \leftrightarrow \leftrightarrow \rightarrow \rightarrow \rightarrow \rightarrow \downarrow$ & $\uparrow \leftrightarrow \leftrightarrow \rightarrow \rightarrow \rightarrow \rightarrow$ & $\uparrow \hookleftarrow \leftarrow \leftrightarrow \rightarrow \rightarrow \rightarrow \downarrow$ & $\leftrightarrow \rightarrow \rightarrow \rightarrow 1$ \\
\hline & $\overrightarrow{\rightarrow \rightarrow \rightarrow \rightarrow \rightarrow \rightarrow 1}$ & $\begin{array}{l}\uparrow \rightarrow \rightarrow \rightarrow \rightarrow \rightarrow \rightarrow \rightarrow \downarrow \\
\uparrow \uparrow \uparrow \uparrow \uparrow \uparrow \uparrow \uparrow \rightarrow \rightarrow 1\end{array}$ & $\begin{array}{l}\uparrow \leftrightarrow \rightarrow \rightarrow \rightarrow \rightarrow \rightarrow \downarrow \\
\uparrow \leftrightarrow \rightarrow \rightarrow \rightarrow \rightarrow \rightarrow 1\end{array}$ & $\begin{array}{lll}1+\leftrightarrow \rightarrow \rightarrow \rightarrow \rightarrow \rightarrow \mid \\
+\rightarrow \rightarrow \rightarrow \rightarrow \rightarrow \mid\end{array}$ & † & $\uparrow \leftrightarrow \leftrightarrow \leftrightarrow \rightarrow \rightarrow \rightarrow \downarrow$ & 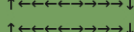 \\
\hline & $\uparrow++++++++0$ & $\uparrow++++++++0$ & $1++++++++0$ & $\begin{array}{l}1++++++++0 \\
0\end{array}$ & $\uparrow+++++++++0$ & $\uparrow++++++++0$ & $1++++++++0$ \\
\hline \multirow[b]{6}{*}{$\gamma=0.99$} & $\rightarrow \rightarrow 1$ & $\rightarrow \rightarrow \rightarrow \rightarrow \rightarrow \rightarrow \rightarrow \rightarrow 1$ & $\rightarrow \rightarrow \rightarrow \rightarrow \rightarrow \rightarrow \rightarrow 1$ & $\rightarrow \rightarrow \rightarrow \rightarrow \rightarrow \rightarrow \rightarrow \rightarrow 1$ & $\rightarrow \rightarrow \rightarrow \rightarrow \rightarrow \rightarrow \rightarrow \rightarrow \downarrow$ & $\rightarrow \rightarrow \rightarrow \rightarrow \rightarrow \rightarrow \rightarrow \rightarrow \downarrow$ & $\rightarrow \rightarrow \rightarrow \rightarrow \rightarrow \rightarrow \rightarrow \mapsto$ \\
\hline & $\rightarrow \rightarrow \downarrow$ & $\uparrow \uparrow \uparrow \rightarrow \rightarrow$ & $\uparrow \uparrow \uparrow \uparrow \uparrow \uparrow \uparrow \rightarrow \rightarrow \downarrow$ & $\uparrow \uparrow \uparrow \uparrow \uparrow \uparrow \uparrow \uparrow \uparrow \downarrow$ & $\uparrow \uparrow \uparrow \uparrow \uparrow \uparrow \uparrow \uparrow \rightarrow \downarrow$ & $\uparrow \uparrow \uparrow \uparrow \uparrow \uparrow \uparrow \uparrow \rightarrow \downarrow$ & $\uparrow \uparrow \uparrow \uparrow \uparrow \uparrow \uparrow \uparrow \rightarrow \downarrow$ \\
\hline & $\rightarrow 1$ & $\uparrow \uparrow \rightarrow \rightarrow$ & $\uparrow+\uparrow \uparrow \uparrow \rightarrow \rightarrow \rightarrow \downarrow$ & $\uparrow \hookleftarrow+\uparrow \uparrow \uparrow \rightarrow \rightarrow \downarrow$ & $\uparrow \leftrightarrow \leftarrow \leftrightarrow \rightarrow \rightarrow \rightarrow \downarrow$ & $\uparrow \leftrightarrow \leftrightarrow \leftrightarrow \rightarrow \rightarrow \rightarrow \downarrow$ & $\uparrow \leftrightarrow \leftrightarrow \leftrightarrow \rightarrow \rightarrow \rightarrow \downarrow$ \\
\hline & $\rightarrow \rightarrow 1$ & $\uparrow \leftrightarrow \rightarrow \rightarrow$ & $\uparrow \leftrightarrow \uparrow \rightarrow \rightarrow \rightarrow \rightarrow \downarrow$ & $\uparrow \leftrightarrow \leftrightarrow \rightarrow \rightarrow \rightarrow \downarrow$ & $\uparrow \leftrightarrow \leftarrow \leftarrow \rightarrow \rightarrow \rightarrow \downarrow$ & $\uparrow \leftrightarrow \leftrightarrow \leftrightarrow \rightarrow \rightarrow \rightarrow \downarrow$ & $\uparrow \hookleftarrow \leftrightarrow \leftrightarrow \rightarrow \rightarrow \rightarrow \downarrow$ \\
\hline & 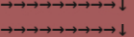 & $\begin{array}{l}\uparrow \leftrightarrow \uparrow \uparrow \rightarrow \rightarrow \rightarrow 1 \\
\uparrow \leftrightarrow \uparrow \uparrow \uparrow \uparrow \rightarrow \rightarrow 1\end{array}$ & $\begin{array}{l}1 \leftrightarrow \leftrightarrow \rightarrow \rightarrow \rightarrow \rightarrow \rightarrow 1 \\
+\leftrightarrow \mapsto \rightarrow \rightarrow \rightarrow \rightarrow 1\end{array}$ & $\begin{array}{l}1 \leftrightarrow+\rightarrow \rightarrow \rightarrow \rightarrow \rightarrow 1 \\
1+\rightarrow \rightarrow \rightarrow \rightarrow \rightarrow 1\end{array}$ & $\uparrow \leftrightarrow \leftrightarrow \leftrightarrow \rightarrow \rightarrow \rightarrow \downarrow$ & $\uparrow \hookleftarrow \leftrightarrow \leftrightarrow \rightarrow \rightarrow \rightarrow \downarrow$ & $\uparrow \leftrightarrow \leftrightarrow \leftrightarrow-$ \\
\hline & $\uparrow++++++++0$ & $\uparrow++++++++0$ & $1++++++++0$ & $1++++++++0$ & $\mathrm{~T}++++++++0$ & $\uparrow++++++++0$ & $1++++++++0$ \\
\hline
\end{tabular}

\begin{tabular}{|c|c|c|c|c|c|c|c|}
\hline $10 \times 7(-)$ & $\mathrm{k}=-0.99$ & $\mathrm{k}=-0.8$ & $\mathrm{k}=-0.5$ & $\mathrm{k}=0$ & $\mathrm{k}=0.5$ & $\mathrm{k}=0.8$ & $\mathrm{k}=0.99$ \\
\hline \multirow[b]{7}{*}{$\gamma=0.6$} & $\rightarrow \rightarrow 1$ & $\rightarrow \rightarrow \rightarrow \rightarrow \rightarrow \rightarrow \downarrow$ & $\rightarrow \rightarrow \rightarrow \rightarrow \rightarrow \rightarrow \downarrow$ & $\rightarrow \rightarrow \rightarrow \rightarrow \rightarrow \rightarrow \rightarrow 1$ & $\rightarrow \rightarrow \rightarrow \rightarrow \rightarrow \rightarrow \rightarrow \rightarrow \downarrow \downarrow$ & $\rightarrow \rightarrow \rightarrow \rightarrow \rightarrow \rightarrow \rightarrow \rightarrow \rightarrow \downarrow$ & $\rightarrow \rightarrow \rightarrow \rightarrow \rightarrow \rightarrow \rightarrow \rightarrow \downarrow$ \\
\hline & $\uparrow \rightarrow \rightarrow \rightarrow \rightarrow \rightarrow \rightarrow \downarrow$ & $\rightarrow \rightarrow \rightarrow \rightarrow \rightarrow \rightarrow \rightarrow \rightarrow$ & $\uparrow \uparrow \uparrow \uparrow \rightarrow \rightarrow \rightarrow \rightarrow \rightarrow \downarrow$ & $\uparrow \uparrow \uparrow \uparrow \uparrow \uparrow \uparrow \rightarrow \rightarrow \downarrow$ & $\uparrow \uparrow \uparrow \uparrow \uparrow \uparrow \uparrow \uparrow \rightarrow \downarrow$ & $\uparrow \uparrow \uparrow \uparrow \uparrow \uparrow \uparrow \uparrow \rightarrow \downarrow$ & $\uparrow \uparrow \uparrow \uparrow \uparrow \uparrow \uparrow \uparrow \rightarrow \downarrow$ \\
\hline & $\rightarrow \rightarrow \rightarrow \rightarrow \rightarrow \rightarrow \rightarrow \rightarrow \downarrow$ & $\rightarrow \rightarrow \rightarrow \rightarrow \rightarrow \rightarrow \rightarrow \rightarrow \rightarrow \downarrow$ & $\uparrow \rightarrow \rightarrow \rightarrow \rightarrow \rightarrow \rightarrow \rightarrow \downarrow$ & $\uparrow \mapsto \uparrow \uparrow \rightarrow \rightarrow \rightarrow \rightarrow 1$ & $\uparrow+\uparrow \uparrow \uparrow \uparrow \rightarrow \rightarrow \rightarrow \downarrow$ & $\uparrow \leftarrow \uparrow \uparrow \uparrow \uparrow \rightarrow \rightarrow \rightarrow \downarrow$ & $\uparrow \leftrightarrow \uparrow \uparrow \uparrow \uparrow \rightarrow \rightarrow \downarrow$ \\
\hline & $\rightarrow \rightarrow \rightarrow \rightarrow \rightarrow \rightarrow \rightarrow \rightarrow \downarrow$ & $\rightarrow \rightarrow \rightarrow \rightarrow \rightarrow \rightarrow \rightarrow \rightarrow \downarrow$ & $\uparrow \rightarrow \rightarrow \rightarrow \rightarrow \rightarrow \rightarrow \rightarrow \downarrow$ & $\uparrow \mapsto \uparrow \rightarrow \rightarrow \rightarrow \rightarrow \rightarrow \downarrow$ & $\uparrow \mapsto \uparrow \uparrow \rightarrow \rightarrow \rightarrow \rightarrow \downarrow$ & $\uparrow \leftrightarrow \leftrightarrow \rightarrow \rightarrow \rightarrow \rightarrow \downarrow$ & $\uparrow \leftrightarrow \leftrightarrow \leftrightarrow \rightarrow \rightarrow \rightarrow \downarrow$ \\
\hline & $\rightarrow \rightarrow \rightarrow \rightarrow \rightarrow \rightarrow \rightarrow \rightarrow \rightarrow \downarrow$ & $\rightarrow \rightarrow \rightarrow \rightarrow \rightarrow \rightarrow \rightarrow \rightarrow \downarrow$ & $\uparrow \rightarrow \rightarrow \rightarrow \rightarrow \rightarrow \rightarrow \rightarrow 1$ & $\uparrow \leftrightarrow \rightarrow \rightarrow \rightarrow \rightarrow \rightarrow 1$ & $\uparrow \leftrightarrow \leftrightarrow \rightarrow \rightarrow \rightarrow \rightarrow \downarrow$ & $\uparrow \leftrightarrow \leftrightarrow \rightarrow \rightarrow \rightarrow \rightarrow \downarrow$ & $\uparrow \leftrightarrow \leftrightarrow \leftrightarrow \rightarrow \rightarrow \rightarrow \downarrow$ \\
\hline & $\rightarrow \rightarrow \rightarrow \rightarrow \rightarrow \rightarrow \rightarrow \rightarrow \downarrow$ & $\uparrow \rightarrow \rightarrow \rightarrow \rightarrow \rightarrow \rightarrow \downarrow$ & $\uparrow \rightarrow \rightarrow \rightarrow \rightarrow \rightarrow \rightarrow 1$ & $\uparrow \leftrightarrow \rightarrow \rightarrow \rightarrow \rightarrow \rightarrow 1$ & $\uparrow \leftrightarrow \leftrightarrow \rightarrow \rightarrow \rightarrow \rightarrow 1$ & $\uparrow \hookleftarrow \leftarrow \rightarrow \rightarrow \rightarrow \rightarrow \downarrow$ & $\uparrow \leftrightarrow \leftrightarrow \leftrightarrow \rightarrow \rightarrow \rightarrow \downarrow$ \\
\hline & $\uparrow++++++++0$ & $\uparrow++++++++0$ & $\uparrow++++++++0$ & $\uparrow++++++++0$ & $\uparrow++++++++0$ & $\uparrow+++++++++0$ & $1++++++++0$ \\
\hline \multirow[b]{7}{*}{$\gamma=0.7$} & $\rightarrow \rightarrow \rightarrow \rightarrow \rightarrow \rightarrow \downarrow \downarrow \downarrow$ & $\rightarrow \rightarrow \rightarrow \rightarrow \rightarrow \rightarrow \rightarrow \rightarrow \rightarrow 1$ & $\rightarrow \rightarrow \rightarrow \rightarrow \rightarrow \rightarrow \rightarrow \rightarrow \rightarrow \downarrow$ & $\rightarrow \rightarrow \rightarrow \rightarrow \rightarrow \rightarrow \rightarrow \rightarrow \rightarrow 1$ & $\rightarrow \rightarrow \rightarrow \rightarrow \rightarrow \rightarrow \rightarrow \rightarrow \rightarrow \downarrow$ & $\rightarrow \rightarrow \rightarrow \rightarrow \rightarrow \rightarrow \rightarrow \rightarrow \rightarrow \downarrow$ & $\rightarrow \rightarrow \rightarrow \rightarrow \rightarrow \rightarrow \rightarrow \rightarrow \mid$ \\
\hline & $\rightarrow \rightarrow \rightarrow \rightarrow \rightarrow \rightarrow \rightarrow \rightarrow \downarrow$ & $\rightarrow \rightarrow \rightarrow \rightarrow \rightarrow \rightarrow \rightarrow \rightarrow \downarrow$ & $\uparrow \uparrow \uparrow \uparrow \uparrow \rightarrow \rightarrow \rightarrow \rightarrow \downarrow$ & $\uparrow \uparrow \uparrow \uparrow \uparrow \uparrow \uparrow \rightarrow \rightarrow \downarrow$ & $\uparrow \uparrow \uparrow \uparrow \uparrow \uparrow \uparrow \uparrow \rightarrow \downarrow$ & $\uparrow \uparrow \uparrow \uparrow \uparrow \uparrow \uparrow \uparrow \uparrow \rightarrow \downarrow$ & $\uparrow \uparrow \uparrow \uparrow \uparrow \uparrow \uparrow \uparrow \rightarrow \downarrow$ \\
\hline & & $\rightarrow \rightarrow \rightarrow \rightarrow \rightarrow \rightarrow 1$ & $\uparrow \uparrow \rightarrow \rightarrow \rightarrow \rightarrow \rightarrow \rightarrow \downarrow$ & $\uparrow \mapsto \uparrow \uparrow \uparrow \rightarrow \rightarrow \rightarrow \rightarrow 1$ & $\uparrow \mapsto \uparrow \uparrow \uparrow \uparrow \rightarrow \rightarrow \rightarrow \downarrow$ & $\uparrow \leftarrow \uparrow \uparrow \uparrow \uparrow \rightarrow \rightarrow \rightarrow \downarrow$ & $\downarrow \leftrightarrow \leftrightarrow \rightarrow \rightarrow \rightarrow \rightarrow \downarrow$ \\
\hline & & $\rightarrow \rightarrow \rightarrow \rightarrow \rightarrow \rightarrow \rightarrow 1$ & $\uparrow \leftrightarrow \rightarrow \rightarrow \rightarrow \rightarrow \rightarrow \downarrow$ & $\uparrow \leftrightarrow \uparrow \rightarrow \rightarrow \rightarrow \rightarrow$ & $\uparrow \mapsto \leftrightarrow \leftrightarrow \rightarrow \rightarrow \rightarrow \downarrow$ & $\uparrow \leftrightarrow \leftrightarrow \rightarrow \rightarrow \rightarrow \rightarrow \downarrow$ & $\uparrow \leftrightarrow \leftrightarrow \leftrightarrow \rightarrow \rightarrow \rightarrow \downarrow$ \\
\hline & & $\rightarrow \rightarrow \rightarrow \rightarrow \rightarrow \rightarrow \rightarrow \downarrow$ & $\uparrow \leftrightarrow \rightarrow \rightarrow \rightarrow \rightarrow \rightarrow \rightarrow 1$ & $\uparrow \leftrightarrow \leftrightarrow \rightarrow \rightarrow \rightarrow \rightarrow 1$ & $\uparrow \leftrightarrow \leftrightarrow \rightarrow \rightarrow \rightarrow \rightarrow 1$ & $\uparrow \hookleftarrow \leftrightarrow \rightarrow \rightarrow \rightarrow \mapsto$ & $\uparrow \leftrightarrow \leftrightarrow \leftrightarrow \rightarrow \rightarrow \rightarrow \rightarrow$ \\
\hline & $\rightarrow \rightarrow \rightarrow \rightarrow \rightarrow$ & $\uparrow \uparrow \uparrow \rightarrow \rightarrow \rightarrow \rightarrow \rightarrow \downarrow$ & $\uparrow \leftrightarrow \rightarrow \rightarrow \rightarrow \rightarrow \rightarrow \downarrow$ & $\mapsto \leftrightarrow \leftrightarrow \rightarrow \rightarrow \rightarrow \rightarrow 1$ & $\uparrow \mapsto \leftrightarrow \rightarrow \rightarrow \rightarrow \downarrow$ & $\uparrow \hookleftarrow \leftrightarrow \rightarrow \rightarrow \rightarrow \rightarrow 1$ & $\uparrow \leftrightarrow \leftrightarrow \rightarrow \rightarrow \rightarrow \rightarrow \downarrow$ \\
\hline & $1++++++++0$ & $\uparrow++++++++0$ & $\uparrow++++++++0$ & $1++++++++0$ & $\uparrow++++++++0$ & $\uparrow++++++++0$ & $1++++++++0$ \\
\hline \multirow[b]{7}{*}{$\gamma=0.8$} & $\rightarrow \rightarrow \rightarrow \rightarrow 11111 \downarrow$ & $\rightarrow \rightarrow \rightarrow \rightarrow \rightarrow \rightarrow \rightarrow \rightarrow \rightarrow \mid$ & $\rightarrow \rightarrow \rightarrow \rightarrow \rightarrow \rightarrow \rightarrow \rightarrow$ & $\rightarrow \rightarrow \rightarrow \rightarrow \rightarrow \rightarrow \rightarrow \rightarrow 1$ & $\rightarrow \rightarrow \rightarrow \rightarrow \rightarrow \rightarrow \rightarrow \rightarrow \rightarrow 1$ & $\rightarrow \rightarrow \rightarrow \rightarrow \rightarrow \rightarrow \rightarrow \rightarrow \perp$ & $\rightarrow \rightarrow \rightarrow \rightarrow \rightarrow \rightarrow \rightarrow \rightarrow \rightarrow \downarrow$ \\
\hline & $\rightarrow \rightarrow \rightarrow 1$ & $\rightarrow \rightarrow \rightarrow \rightarrow \rightarrow \rightarrow \rightarrow \downarrow$ & $\uparrow \uparrow \uparrow \uparrow \uparrow \rightarrow \rightarrow \rightarrow \rightarrow \downarrow$ & $\uparrow \uparrow \uparrow \uparrow \uparrow \uparrow \uparrow \rightarrow \rightarrow \downarrow$ & $\uparrow \uparrow \uparrow \uparrow \uparrow \uparrow \uparrow \uparrow \uparrow \rightarrow \downarrow$ & $\uparrow \uparrow \uparrow \uparrow \uparrow \uparrow \uparrow \uparrow \rightarrow \downarrow$ & $\uparrow \uparrow \uparrow \uparrow \uparrow \uparrow \uparrow \uparrow \rightarrow \downarrow$ \\
\hline & & & $\uparrow \uparrow \uparrow \rightarrow \rightarrow \rightarrow \rightarrow \rightarrow \downarrow$ & $\uparrow \uparrow \uparrow \uparrow \uparrow \rightarrow \rightarrow \rightarrow \downarrow$ & $\uparrow+\uparrow \uparrow \uparrow \uparrow \rightarrow \rightarrow \rightarrow \downarrow$ & $\uparrow \leftrightarrow \uparrow \uparrow \uparrow \uparrow \rightarrow \rightarrow \downarrow$ & $\uparrow \leftrightarrow \leftrightarrow \leftrightarrow \rightarrow \rightarrow \rightarrow \downarrow$ \\
\hline & & $\rightarrow \rightarrow \rightarrow$ & $\uparrow \leftrightarrow \rightarrow \rightarrow \rightarrow \rightarrow \downarrow$ & $\uparrow \leftarrow \leftarrow \uparrow \rightarrow \rightarrow \rightarrow \rightarrow \rightarrow \downarrow$ & $\uparrow \hookleftarrow \leftrightarrow \rightarrow \rightarrow \rightarrow \rightarrow \downarrow$ & $\uparrow \leftarrow \leftarrow \leftrightarrow \rightarrow \rightarrow \rightarrow \rightarrow \downarrow$ & $\uparrow \leftrightarrow \leftrightarrow \rightarrow \rightarrow \rightarrow \downarrow$ \\
\hline & & $\uparrow \rightarrow \rightarrow \rightarrow \rightarrow$ & $\uparrow \leftrightarrow \leftrightarrow \rightarrow \rightarrow \rightarrow \rightarrow 1$ & $\uparrow \leftrightarrow \leftrightarrow \leftrightarrow \rightarrow \rightarrow \rightarrow \downarrow$ & $\uparrow \leftrightarrow \leftrightarrow \leftrightarrow \rightarrow \rightarrow \rightarrow \downarrow$ & $\uparrow \leftrightarrow \leftrightarrow \rightarrow \rightarrow \rightarrow \rightarrow \downarrow$ & $\uparrow \leftrightarrow \leftrightarrow \rightarrow \rightarrow \rightarrow \rightarrow \downarrow$ \\
\hline & & $\uparrow \uparrow \uparrow \uparrow \uparrow \rightarrow \rightarrow \rightarrow \downarrow$ & $\uparrow \leftrightarrow \leftrightarrow \rightarrow \rightarrow \rightarrow \downarrow$ & $\uparrow \leftrightarrow \leftrightarrow \leftrightarrow \rightarrow \rightarrow \rightarrow \downarrow$ & $\uparrow \leftrightarrow \leftrightarrow \leftrightarrow \rightarrow \rightarrow \rightarrow \downarrow$ & $\uparrow \leftrightarrow \leftrightarrow \rightarrow \rightarrow \rightarrow \rightarrow \downarrow$ & $\uparrow \leftrightarrow \leftrightarrow \rightarrow \rightarrow \rightarrow \downarrow$ \\
\hline & $1++++++++0$ & $\uparrow++++++++0$ & $\uparrow++++++++0$ & $1++++++++0$ & †+++++++++o & $\uparrow+++++++++0$ & $1++++++++0$ \\
\hline \multirow[b]{7}{*}{$\gamma=0.9$} & $\rightarrow \rightarrow \downarrow \downarrow \downarrow \downarrow \downarrow \downarrow \downarrow \downarrow$ & $\rightarrow \rightarrow \rightarrow \rightarrow \rightarrow \rightarrow \rightarrow \rightarrow \rightarrow \mid$ & $\rightarrow \rightarrow \rightarrow \rightarrow \rightarrow \rightarrow \rightarrow \rightarrow \rightarrow \mid$ & $\rightarrow \rightarrow \rightarrow \rightarrow \rightarrow \rightarrow \rightarrow \rightarrow \rightarrow \mid$ & $\rightarrow \rightarrow \rightarrow \rightarrow \rightarrow \rightarrow \rightarrow \rightarrow \rightarrow \mid$ & $\rightarrow \rightarrow \rightarrow \rightarrow \rightarrow \rightarrow \rightarrow \rightarrow \rightarrow \mid$ & $\rightarrow \rightarrow \rightarrow \rightarrow \rightarrow \rightarrow \rightarrow \rightarrow \rightarrow 1$ \\
\hline & & $\uparrow \rightarrow \rightarrow \rightarrow \rightarrow \rightarrow \rightarrow \downarrow$ & $\uparrow \uparrow \uparrow \uparrow \uparrow \uparrow \rightarrow \rightarrow \rightarrow \downarrow$ & $\uparrow \uparrow \uparrow \uparrow \uparrow \uparrow \uparrow \uparrow \rightarrow \downarrow$ & $\uparrow \uparrow \uparrow \uparrow \uparrow \uparrow \uparrow \uparrow \rightarrow \downarrow$ & $\uparrow \uparrow \uparrow \uparrow \uparrow \uparrow \uparrow \uparrow \rightarrow \downarrow$ & $\uparrow \uparrow \uparrow \tau \uparrow \uparrow \uparrow \uparrow \rightarrow \downarrow$ \\
\hline & & $\uparrow \rightarrow \rightarrow \rightarrow \rightarrow \rightarrow \rightarrow \downarrow$ & $\uparrow+\uparrow \uparrow \uparrow \rightarrow \rightarrow \rightarrow \downarrow$ & $\uparrow+\uparrow \uparrow \uparrow \uparrow \rightarrow \rightarrow \rightarrow \downarrow$ & $\uparrow \leftarrow \leftarrow \uparrow \uparrow \uparrow \rightarrow \rightarrow \downarrow$ & $\uparrow \hookleftarrow \hookleftarrow \leftrightarrow \rightarrow \rightarrow \rightarrow \downarrow$ & $\uparrow \leftrightarrow \leftrightarrow \rightarrow \rightarrow \rightarrow \downarrow$ \\
\hline & $\rightarrow \rightarrow$ & $\uparrow \rightarrow \rightarrow \rightarrow \rightarrow \rightarrow \rightarrow \downarrow$ & $\uparrow \leftrightarrow \leftrightarrow \rightarrow \rightarrow \rightarrow \rightarrow 1$ & $\uparrow \leftrightarrow \leftrightarrow \rightarrow \rightarrow \rightarrow 1$ & $\uparrow \leftrightarrow \leftrightarrow \rightarrow \rightarrow \rightarrow \rightarrow 1$ & $\uparrow \leftrightarrow \leftrightarrow \leftrightarrow \rightarrow \rightarrow \rightarrow 1$ & $\uparrow \leftrightarrow \leftrightarrow \rightarrow \rightarrow \rightarrow 1$ \\
\hline & $\rightarrow \rightarrow$ & $\uparrow \rightarrow \rightarrow \rightarrow \rightarrow \rightarrow \rightarrow \rightarrow \rightarrow \downarrow$ & $\uparrow \leftrightarrow \rightarrow \rightarrow \rightarrow \rightarrow \rightarrow \downarrow$ & $\uparrow \leftrightarrow \leftrightarrow \rightarrow \rightarrow \rightarrow 1$ & $\uparrow \leftrightarrow \leftrightarrow \rightarrow \rightarrow \rightarrow \rightarrow \downarrow$ & $\uparrow \leftrightarrow \leftrightarrow \leftrightarrow \rightarrow \rightarrow \rightarrow \downarrow$ & $\uparrow \leftrightarrow \leftrightarrow \rightarrow \rightarrow \rightarrow \rightarrow \downarrow$ \\
\hline & $\rightarrow \rightarrow \rightarrow \rightarrow \rightarrow \rightarrow \rightarrow \rightarrow \rightarrow 1$ & $\uparrow \uparrow \uparrow \uparrow \uparrow \uparrow \uparrow \rightarrow \rightarrow \downarrow$ & $\uparrow \leftrightarrow \leftrightarrow \rightarrow \rightarrow \rightarrow \rightarrow \downarrow$ & $\uparrow \leftrightarrow \leftrightarrow \rightarrow \rightarrow \rightarrow+1$ & $\uparrow \leftrightarrow \leftrightarrow \leftrightarrow \rightarrow \rightarrow \rightarrow \downarrow$ & $\uparrow \leftrightarrow \leftrightarrow \leftrightarrow \rightarrow \rightarrow \rightarrow \downarrow$ & $\uparrow \leftrightarrow \leftrightarrow \rightarrow \rightarrow \rightarrow \rightarrow 1$ \\
\hline & $1++++++++0$ & $1++++++++0$ & $\uparrow++++++++0$ & $\uparrow++++++++0$ & $\uparrow++++++++0$ & $\uparrow++++++++0$ & $1++++++++0$ \\
\hline \multirow[b]{7}{*}{$\gamma=0.99$} & 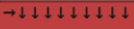 & $\rightarrow \rightarrow \rightarrow \rightarrow \rightarrow \rightarrow \rightarrow \rightarrow \rightarrow \downarrow$ & $\rightarrow \rightarrow \rightarrow \rightarrow \rightarrow \rightarrow \rightarrow \rightarrow \rightarrow \downarrow$ & $\rightarrow \rightarrow \rightarrow \rightarrow \rightarrow \rightarrow \rightarrow \rightarrow \rightarrow \mid$ & $\rightarrow \rightarrow \rightarrow \rightarrow \rightarrow \rightarrow \rightarrow \rightarrow \rightarrow \downarrow$ & $\rightarrow \rightarrow \rightarrow \rightarrow \rightarrow \rightarrow \rightarrow \rightarrow \rightarrow \downarrow$ & $\rightarrow \rightarrow \rightarrow \rightarrow \rightarrow \rightarrow \rightarrow \rightarrow \rightarrow$ \\
\hline & & $\uparrow \uparrow \uparrow \rightarrow \rightarrow \rightarrow \rightarrow \rightarrow \rightarrow \downarrow$ & $\uparrow \uparrow \uparrow \uparrow \uparrow \uparrow \uparrow \rightarrow \rightarrow \downarrow$ & $\uparrow \uparrow \uparrow \uparrow \uparrow \uparrow \uparrow \uparrow \rightarrow \downarrow$ & $\uparrow \uparrow \uparrow \uparrow \uparrow \uparrow \uparrow \uparrow \uparrow \rightarrow \downarrow$ & $\uparrow \uparrow \uparrow \uparrow \uparrow \uparrow \uparrow \uparrow \uparrow \rightarrow \downarrow$ & $\uparrow \uparrow \uparrow \uparrow \uparrow \uparrow \uparrow \uparrow \uparrow \rightarrow \downarrow$ \\
\hline & $\rightarrow \rightarrow$ & $\uparrow \uparrow \rightarrow \rightarrow \rightarrow \rightarrow \rightarrow \rightarrow \rightarrow \downarrow$ & $\uparrow \leftrightarrow \uparrow \uparrow \uparrow \rightarrow \rightarrow \rightarrow \downarrow$ & $\uparrow \leftrightarrow \uparrow \uparrow \uparrow \rightarrow \rightarrow \rightarrow \downarrow$ & $\uparrow \hookleftarrow \leftrightarrow \leftrightarrow \rightarrow \rightarrow \rightarrow \downarrow$ & $\uparrow \leftrightarrow \leftrightarrow \mapsto \rightarrow \rightarrow \downarrow$ & $\uparrow \leftrightarrow \leftrightarrow \leftrightarrow \rightarrow \rightarrow \rightarrow \downarrow$ \\
\hline & & $\uparrow \leftrightarrow \rightarrow \rightarrow \rightarrow \rightarrow \rightarrow \rightarrow \downarrow$ & $\uparrow \leftarrow+\uparrow \rightarrow \rightarrow \rightarrow \rightarrow \downarrow$ & $\uparrow \leftrightarrow \leftrightarrow \rightarrow \rightarrow \rightarrow 1$ & $\uparrow \hookleftarrow \hookleftarrow \leftrightarrow \rightarrow \rightarrow \rightarrow \downarrow$ & $\uparrow \hookleftarrow \leftrightarrow \mapsto \rightarrow \rightarrow \rightarrow \downarrow$ & $\uparrow \leftrightarrow \leftrightarrow \leftrightarrow \rightarrow \rightarrow \rightarrow \downarrow$ \\
\hline & & $\uparrow \mapsto \uparrow \uparrow \rightarrow \rightarrow \rightarrow \downarrow$ & $\uparrow \leftrightarrow \leftrightarrow \leftrightarrow \rightarrow \rightarrow \rightarrow \downarrow$ & $\uparrow \leftrightarrow \leftrightarrow \rightarrow \rightarrow \rightarrow \downarrow$ & $\uparrow \leftrightarrow \leftrightarrow \leftrightarrow \rightarrow \rightarrow \mapsto$ & $\uparrow \leftrightarrow \leftrightarrow \leftrightarrow \rightarrow \rightarrow \downarrow$ & $\uparrow \leftrightarrow \leftrightarrow \leftrightarrow \rightarrow \rightarrow \mapsto$ \\
\hline & & $\uparrow \leftrightarrow \uparrow \uparrow \uparrow \uparrow \rightarrow \neg$ & $\uparrow \leftrightarrow \leftrightarrow \rightarrow \rightarrow \rightarrow \downarrow$ & $\uparrow \leftrightarrow \leftrightarrow \rightarrow \rightarrow \rightarrow \downarrow$ & $\uparrow \hookleftarrow \leftrightarrow \leftrightarrow \rightarrow \rightarrow \rightarrow$ & $\uparrow \hookleftarrow \leftarrow \leftrightarrow \rightarrow \rightarrow \rightarrow \downarrow$ & $\uparrow \leftrightarrow \leftrightarrow \leftrightarrow \leftrightarrow \rightarrow \rightarrow \downarrow$ \\
\hline & ++++++++0 & $\uparrow++++++++0$ & $\uparrow++++++++0$ & $\uparrow++++++++0$ & $\uparrow++++++++0$ & $\uparrow++++++++0$ & $\uparrow++++++++0$ \\
\hline
\end{tabular}

O fator de desconto por sua vez tem o papel de garantir que a convergência ocorra e tem influência no tempo necessário para convergência, porém de forma mais tênue se 

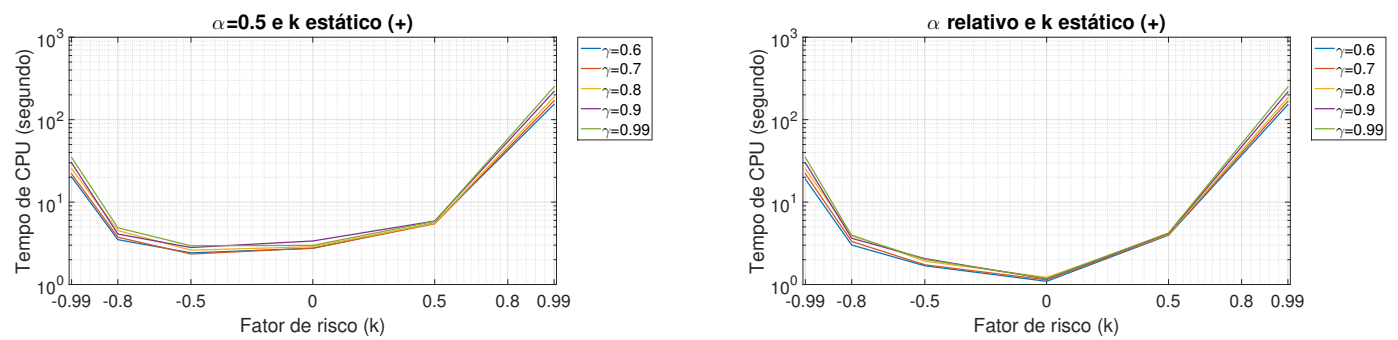

Figura 1. Tempo de convergência em um rio de tamanho $10 \times 7$ no cenário de recompensas (+).
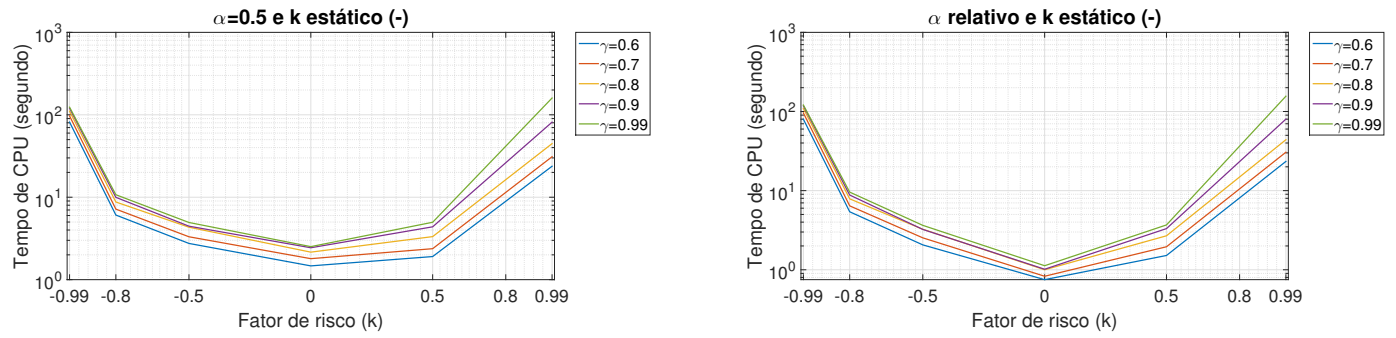

Figura 2. Tempo de convergência em um rio de tamanho $10 \times 7$ no cenário de recompensas (-).

comparado ao impacto do fator de risco no tempo. Quanto maior o desconto, maior o tempo necessário para convergência.

Na Figura 3 é mostrado o tempo para diferentes tamanhos de grid considerando $\gamma=0.99, \alpha$ relativo nos cenários de recompensas $(+)$ e $(-)$ para $k=-0.99$ (lado esquerdo da figura) e $k=0.99$ (lado direito da figura). A figura mostra que o tempo de processamento depende do tamanho do problema, quanto maior a quantidade de estados, maior o tempo necessário para convergência. Note que para $k=-0.99$, a adoção de um cenário $(+)$ pode reduzir o tempo de convergência do algoritmo quando comparado com o cenário $(-)$, o contrário ocorre para $k=0.99$.
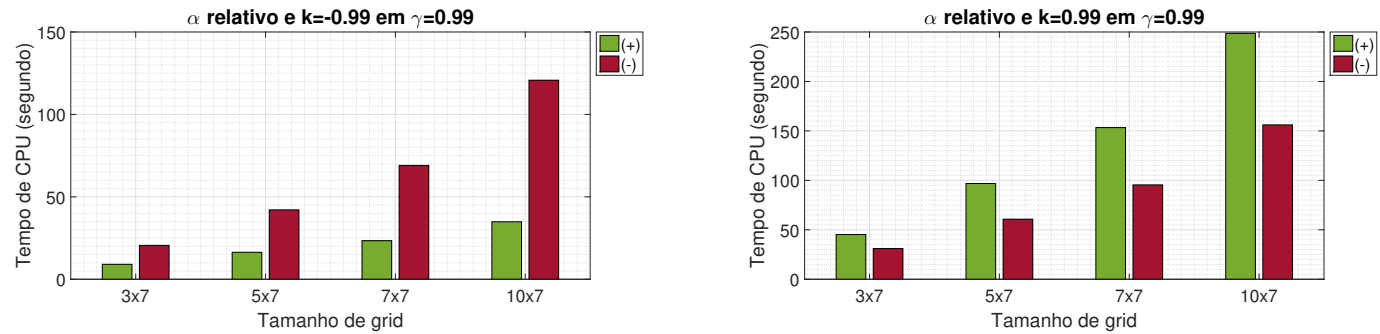

Figura 3. Tempo de processamento por tamanho de grid e cenário de recompensa para $k=-0.99$ e $k=0.99$.

\subsection{Convergência $\operatorname{com} \alpha=1$}

A convergência do algoritmo ocorre tanto no cenário $(+)$ quanto $(-) \operatorname{com} \alpha=0.5$ e $\alpha$ relativo a $k$. Também foram feitos testes $\operatorname{com} \alpha=1$ e para problemas com um número pequeno de estados o algoritmo converge muito rápido. Contudo vale ressaltar que em problemas com um número de estados elevado no cenário $(+)$ e um fator de desconto alto 
( $\gamma$ próximo de 1), o algoritmo não consegue convergir, sendo que os valores crescem até o infinito.

\section{Considerações Finais}

Neste trabalho, foi analisado de forma empírica o impacto da escolha do fator de risco e o fator de desconto na política e no tempo de convergência do algoritmo de Iteração de Valor de [Mihatsch and Neuneier 2002]. O algoritmo de Iteração de Valor Sensível a Risco consegue encontrar políticas sensíveis ao risco conforme o fator de risco escolhido, tanto para aversão $(k>0)$ quanto propensão $(k<0)$ ao risco. Além disso, a característica de neutralidade é mantida quando $k=0$ sendo equivalente ao algoritmo de Iteração de Valor clássico. Nesta abordagem é necessário a escolha de um valor de risco $k$ arbitrário coerente ao domínio de aplicação, para garantir que ocorra a ponderação esperada e permita a construção de uma política $\pi$ com a sensibilidade ao risco desejada.

Foram realizados experimentos no domínio de Travessia do Rio com diferentes tamanhos de grid em dois cenários de recompensas distintos: recompensas positivas acumuladas e recompensas negativas acumuladas. $\mathrm{O}$ fator de desconto tem o papel de atenuar as atitudes se o desconto for pequeno ou realçar as atitudes se o desconto for grande. No limite de $k(k=-0.99$ para propensão extrema ao risco e $k=0.99$ para aversão extrema ao risco), embora o algoritmo encontre políticas com as respectivas atitudes de risco esperadas, o algoritmo demora mais tempo para convergir. Para $k=-0.99$, a adoção de um cenário $(+)$ pode reduzir o tempo de convergência do algoritmo quando comparado com o cenário $(-)$, o contrário ocorre para $k=0.99$.

Os experimentos mostram que o tempo de processamento está diretamente relacionado ao fator de risco escolhido, o fator de desconto $\gamma$, o fator $\alpha$ e o tamanho da instância. Enquanto o fator de desconto tem uma influência fraca no tempo de convergência, o uso de um $\alpha$ dependente de $k$ traz bastante ganho no desempenho do algoritmo reduzindo o número de iterações necessárias para o algoritmo convergir.

Em trabalhos futuros pretende-se analisar o uso de $\alpha$ variando durante as iterações do algoritmo, começando com $\alpha=1$; bem como alterar o fator de risco durante $\mathrm{o}$ algoritmo, por exemplo começar com $k=0$ e incrementar (decrementar) o fator de risco durante as iterações até chegar no valor $k$ desejado.

\section{Agradecimentos}

Os autores agradecem à Capes pela concessão da bolsa de mestrado para as atividades de pesquisa e à FAPESP pelo apoio financeiro (processo \#2015/01587-0).

\section{Referências}

[Bellman 1957] Bellman, R. (1957). A Markovian decision process. Indiana Univ. Math. J., 6:679-684.

[Chung and Sobel 1987] Chung, K.-J. and Sobel, M. J. (1987). Discounted mdp's: distribution functions and exponential utility maximization. SIAM J. Control Optim., 25:49-62.

[Denardo and Rothblum 1979] Denardo, E. V. and Rothblum, U. G. (1979). Optimal stopping, exponential utility, and linear programming. Mathematical Programming, 16(1):228-244. 
[Filar et al. 1989] Filar, J. A., Kallenberg, L. C. M., and Lee, H.-M. (1989). Variancepenalized Markov decision processes. Mathematics of Operations Research, 14(1):147-161.

[Filar et al. 1995] Filar, J. A., Krass, D., Ross, K. W., and Ross, K. W. (1995). Percentile performance criteria for limiting average Markov decision processes. IEEE Transactions on Automatic Control, 40(1):2-10.

[Freire 2016] Freire, V. (2016). The role of discount factor in risk sensitive markov decision processes. In 2016 5th Brazilian Conference on Intelligent Systems (BRACIS), pages $480-485$.

[Freire and Delgado 2017] Freire, V. and Delgado, K. V. (2017). GUBS: a utility-based semantic for Goal-Directed Markov Decision Processes. In Sixteenth International Conference on Autonomous Agents \& Multiagent Systems, pages 741-749.

[García and Fernández 2015] García, J. and Fernández, F. (2015). A comprehensive survey on safe reinforcement learning. J. Mach. Learn. Res., 16(1):1437-1480.

[Hou et al. 2014] Hou, P., Yeoh, W., and Varakantham, P. (2014). Revisiting risk-sensitive MDPs: New algorithms and results. In Proceedings of the Twenty-Fourth International Conference on Automated Planning and Scheduling, ICAPS 2014, Portsmouth, New Hampshire, USA, June 21-26, 2014.

[Hou et al. 2016] Hou, P., Yeoh, W., and Varakantham, P. (2016). Solving risk-sensitive POMDPs with and without cost observations. In Proceedings of the Thirtieth AAAI Conference on Artificial Intelligence, February 12-17, 2016, Phoenix, Arizona, USA., pages 3138-3144.

[Howard and Matheson 1972] Howard, R. A. and Matheson, J. E. (1972). Risk-sensitive Markov decision processes. Management science, 18(7):356-369.

[Jaquette 1976] Jaquette, S. C. (1976). A utility criterion for Markov decision processes. Management Science, 23(1):43-49.

[Mihatsch and Neuneier 2002] Mihatsch, O. and Neuneier, R. (2002). Risk-sensitive reinforcement learning. Machine Learning, 49(2):267-290.

[Patek 2001] Patek, S. D. (2001). On terminating markov decision processes with a riskaverse objective function. Automatica, 37(9):1379-1386.

[Puterman 1994] Puterman, M. L. (1994). Markov Decision Processes: Discrete Stochastic Dynamic Programming. John Wiley \& Sons, Inc., New York, NY, USA, 1st edition.

[Rothblum 1984] Rothblum, U. G. (1984). Multiplicative Markov decision chains. Mathematics of Operations Research, 9(1):6-24.

[Shen et al. 2014] Shen, Y., Tobia, M. J., Sommer, T., and Obermayer, K. (2014). Risksensitive reinforcement learning. Neural computation, 26(7):1298-1328.

[Sobel 1982] Sobel, M. J. (1982). The variance of discounted Markov decision processes. Journal of Applied Probability, 19(4):794-802.

[Yu et al. 1998] Yu, S. X., Lin, Y., and Yan, P. (1998). Optimization models for the first arrival target distribution function in discrete time. Journal of Mathematical Analysis and Applications, 225(1):193 - 223. 